KERWIN MORRIS (Adelaide)

Dominik Szynal (Lublin)

\title{
GOODNESS-OF-FIT TESTS USING CHARACTERIZATIONS OF CONTINUOUS DISTRIBUTIONS
}

Abstract. Using characterization conditions of continuous distributions in terms of moments of order statistics and moments of record values we present new goodness-of-fit techniques.

1. Introduction and preliminaries. Let $\left(X_{1}, \ldots, X_{n}\right)$ be a sample from a continuous distribution $F(x)=P[X \leq x], x \in \mathbb{R}$, and let $X_{k: n}$ denote the $k$ th smallest order statistic of the sample. We construct goodness-of-fit tests for continuous distributions using characterizations of distributions via moments of order statistics and moments of record values (cf. [2]-[5], [10]). The results presented extend the tests for uniformity and exponentiality discussed in [6] and [7]. Moreover, we give the proof of statements on tests for exponentiality announced in [7]. We include a theorem on the asymptotic effect of substituting estimators for parameters in the tests proposed here. It can be used, among other things, to construct a test for normality.

(O) Characterizations in terms of moments of order statistics. We use the characterization conditions contained in the following theorems.

TheOREM 1 (cf. [10], [3]). Let $n, k, l$ be given integers such that $n \geq k \geq l \geq 1$. Assume that $G$ is a nondecreasing right-continuous function from $\mathbb{R}$ to $\mathbb{R}$. Then $F(x)=G(x)$ on $I(F)$ (the minimal interval containing the support of $F$ ) and $F$ is continuous on $\mathbb{R}$ iff

$$
\begin{aligned}
\frac{(k-l) !}{(n-l+1) !} E G^{2 l}( & \left.X_{k+1-l: n+1-l}\right) \\
& -\frac{2 k !}{(n+1) !} E G^{l}\left(X_{k+1: n+1}\right)+\frac{(k+l) !}{(n+l+1) !}=0 .
\end{aligned}
$$

2000 Mathematics Subject Classification: Primary 62E10, 62F03.

Key words and phrases: goodness-of-fit tests, characterizations, order statistics, record values, uniform, exponential, Weibull, Pareto, geometric and logarithmic distributions. 
TheOREM 2 (cf. [5]). Under the assumptions of Theorem 1, $F(x)=$ $G(x)$ on $I(F)$ and $F$ is continuous on $\mathbb{R}$ iff

$$
\begin{aligned}
& E G^{l}\left(X_{k+1: n+1}\right)=\frac{(k+l) !(n+1) !}{k !(n+l+1) !} \\
& E G^{2 l}\left(X_{k+1-l: n+1-l}\right)=\frac{(k+l) !(n-l+1) !}{(k-l) !(n+l+1) !} .
\end{aligned}
$$

Note that Theorem 2 is a consequence of Theorem 1, since (1.1) implies $F=G$ implies (1.2) implies (1.1).

Corollary 1. $X \sim F$ and $F$ is continuous iff

$$
E F\left(X_{2: 2}\right)-E F^{2}(X)=\frac{1}{3}
$$

or

$$
E F\left(X_{2: 2}\right)=\frac{2}{3}, \quad E F^{2}(X)=\frac{1}{3} .
$$

In particular:

(a) $X \sim U(\alpha, \beta)$ (uniform distribution), i.e. $F(x)=(x-\alpha) /(\beta-\alpha)$, $\alpha<x<\beta$, iff

$$
E\left[\left(X_{2: 2}-\alpha\right) /(\beta-\alpha)\right]-E[(X-\alpha) /(\beta-\alpha)]^{2}=\frac{1}{3}
$$

or

$$
E\left[\left(X_{2: 2}-\alpha\right) /(\beta-\alpha)\right]=\frac{2}{3}, \quad E[(X-\alpha) /(\beta-\alpha)]^{2}=\frac{1}{3},
$$

(b) $X \sim \operatorname{Exp}(\alpha)$ (exponential distribution), i.e. $F(x)=1-\exp (-\alpha x)$, $x>0, \alpha>0$, iff

$$
E\left(1-\exp \left(-\alpha X_{2: 2}\right)\right)-E(1-\exp (-\alpha X))^{2}=\frac{1}{3}
$$

or

$$
E\left(1-\exp \left(-\alpha X_{2: 2}\right)\right)=\frac{2}{3}, \quad E(1-\exp (-\alpha X))^{2}=\frac{1}{3} .
$$

(R) Characterization conditions in terms of moments of record values. Let $\left\{X_{n}, n \geq 1\right\}$ be a sequence of i.i.d. random variables with cdf $F$ and pdf $f$. For a fixed $k \geq 1$ we define the sequence $U_{k}(1), U_{k}(2), \ldots$ of $k$-(upper) record times of $X_{1}, X_{2}, \ldots$ as follows:

$$
\begin{aligned}
& U_{k}(1)=1, \\
& U_{k}(n)=\min \left\{j>U_{k}(n-1): X_{j: j+k-1}>X_{U_{k}(n-1): U_{k}(n-1)+k-1}\right\}, \\
& n=2,3, \ldots
\end{aligned}
$$

Write

$$
Y_{n}^{(k)}:=X_{U_{k}(n): U_{k}(n)+k-1}, \quad n \geq 1
$$


The sequence $\left\{Y_{n}^{(k)}, n \geq 1\right\}$ is called the sequence of $k$-(upper) record values of the above sequence. For convenience we also take $Y_{0}^{(k)}=0$ and note that $Y_{1}^{(k)}=X_{1: k}=\min \left(X_{1}, \ldots, X_{k}\right)$ (cf. [1]).

We see that for $k=1,2, \ldots$, the sequences $\left\{Y_{n}^{(k)}, n \geq 1\right\}$ of $k$ th record values can be obtained from $\left\{X_{n}, n \geq 1\right\}$ by inspecting successively the samples $X_{1},\left(X_{1}, X_{2}\right),\left(X_{1}, X_{2}, X_{3}\right)$, and so on. For $k=1, Y_{1}^{(1)}=X_{1}$, and the following terms are obtained by looking at the maxima of the successive samples; $Y_{2}^{(1)}$ is the first maximum that exceeds $Y_{1}^{(1)}, Y_{3}^{(1)}$ is the first maximum that exceeds $Y_{2}^{(1)}$, and so on. For $k=2, Y_{1}^{(2)}=\min \left(X_{1}, X_{2}\right)$, and the following terms are obtained by looking at the next-to-largest values in the successive samples: $Y_{2}^{(2)}$ is the first next-to-largest value that exceeds $Y_{1}^{(2)}, Y_{3}^{(2)}$ is the next-to-largest value that exceeds $Y_{2}^{(2)}$, and so on. And generally, $Y_{1}^{(k)}=\min \left(X_{1}, \ldots, X_{k}\right)=X_{1: k}$, and the following $k$ th record values are obtained by looking at the $k$ th largest values in successive samples, i.e., looking at the order statistics $X_{2: k+1}$ from $\left(X_{1}, \ldots, X_{k+1}\right), X_{3: k+2}$ from $\left(X_{1}, \ldots, X_{k+2}\right)$, and so on.

We have the following characterizations.

TheOREm 3 (cf. [4]). Let $\left\{X_{n}, n \geq 1\right\}$ be a sequence of i.i.d. random variables with cdf $F$. Assume that $G$ is a nondecreasing right-continuous function from $\mathbb{R}$ to $(-\infty, 1]$, and let $n, k, l$ be given integers such that $k \geq 1$ and $n \geq l \geq 1$. Then $F(x)=G(x)$ on $I(F)$ iff

$$
\begin{aligned}
k^{2 l}(n-l) ! E\left[-\log \left(1-G\left(Y_{n-l+1}^{(k)}\right)\right)\right]^{2 l} & \\
& -2 n ! k^{l} E\left[-\log \left(1-G\left(Y_{n+1}^{(k)}\right)\right)\right]^{l}+(n+l) !=0 .
\end{aligned}
$$

TheOREM 4 (cf. [5], [4]). Under the assumptions of Theorem $3, F(x)=$ $G(x)$ on $I(F)$ iff

$$
\begin{gathered}
E\left[-\log \left(1-G\left(Y_{n+1}^{(k)}\right)\right)\right]^{l}=\frac{(n+l) !}{n ! k^{l}}, \\
E\left[-\log \left(1-G\left(Y_{n-l+1}^{(k)}\right)\right)\right]^{2 l}=\frac{(n+l) !}{(n-l) ! k^{2 l}} .
\end{gathered}
$$

Following the observation after Theorem 2 we see that Theorem 4 is a consequence of Theorem 3.

Corollary 2. $X \sim F$ and $F$ is continuous iff

$$
E\left[-\log \left(1-F\left(Y_{1}^{(k)}\right)\right)\right]^{2}-\frac{2}{k} E\left[-\log \left(1-F\left(Y_{2}^{(k)}\right)\right)\right]+\frac{2}{k^{2}}=0
$$

or

$$
E\left[-\log \left(1-F\left(Y_{2}^{(k)}\right)\right)\right]=\frac{2}{k}, \quad E\left[-\log \left(1-F\left(Y_{1}^{(k)}\right)\right)\right]^{2}=\frac{2}{k^{2}} .
$$


In particular:

(a) $X \sim U(0,1)$ iff

$$
E\left[-\log \left(1-Y_{1}^{(k)}\right)\right]^{2}-\frac{2}{k} E\left[-\log \left(1-Y_{2}^{(k)}\right)\right]+\frac{2}{k^{2}}=0
$$

or

$$
E\left[-\log \left(1-Y_{2}^{(k)}\right)\right]=\frac{2}{k}, \quad E\left[-\log \left(1-Y_{1}^{(k)}\right)\right]^{2}=\frac{2}{k^{2}},
$$

(b) $X \sim \operatorname{Exp}(\alpha)$ iff

$$
\alpha^{2} E\left(Y_{1}^{(k)}\right)^{2}-\frac{2}{k^{2}} \alpha E\left(Y_{2}^{(k)}\right)+\frac{2}{k^{2}}=0
$$

or

$$
E Y_{2}^{(k)}=\frac{2}{\alpha k}, \quad E\left(Y_{1}^{(k)}\right)^{2}=\frac{2}{\alpha^{2} k^{2}} .
$$

2. Goodness-of-fit tests based on characterizations via moments of order statistics. The cases when parameters of $F$ are specified and unknown will be treated separately.

(A) Parameters of $F$ are specified. First we construct goodness-of-fit tests based on the characterization in (1.1) (see also (1.3)) which we can write in the form

$$
E\left(F\left(X_{2: 2}\right)\right)-\frac{1}{2}\left(E\left(F^{2}\left(X_{1}\right)+F^{2}\left(X_{2}\right)\right)\right)=\frac{1}{3}
$$

where $X_{1}$ and $X_{2}$ are i.i.d. as $X$.

Let $\left(X_{1}, \ldots, X_{2 n}\right)$ be a sample from $F$, where $F$ is continuous and strictly increasing. Define

$$
\begin{aligned}
Y_{j} & =F^{2}\left(X_{2 j-1}\right)+F^{2}\left(X_{2 j}\right), \\
Z_{j} & =F\left(\max \left(X_{2 j-1}, X_{2 j}\right)\right), \quad j=1, \ldots, n .
\end{aligned}
$$

Then $Y_{1}, \ldots, Y_{n}$ are i.i.d. and $Z_{1}, \ldots, Z_{n}$ are i.i.d. Writing $Y:=Y_{1}=$ $F^{2}\left(X_{1}\right)+F^{2}\left(X_{2}\right), Z:=Z_{1}=F\left(\max \left(X_{1}, X_{2}\right)\right)$ we state the following result.

Lemma 1. Under the above assumptions, the density function of $(Y, Z)$ is given by

$$
f(y, z)= \begin{cases}1 / \sqrt{y-z^{2}}, & 0 \leq z \leq 1, z^{2}<y \leq 2 z^{2} \\ 0, & \text { otherwise }\end{cases}
$$

and

$$
E Y=\frac{2}{3}, \operatorname{Var}(Y)=\frac{8}{45}, E Z=\frac{2}{3}, \operatorname{Var}(Z)=\frac{1}{18}, \operatorname{Cov}(Y, Z)=\frac{4}{45} .
$$

Now we define

$$
R_{j}=Z_{j}-\frac{1}{2} Y_{j}, \quad j=1, \ldots, n .
$$


We see that

$$
\begin{aligned}
E R_{j} & =E Z_{j}-\frac{1}{2} E Y_{j}=\frac{1}{3}, \\
\operatorname{Var} R_{j} & =\operatorname{Var} Z_{j}+\frac{1}{4} \operatorname{Var} Y_{j}-\operatorname{Cov}\left(Z_{j}, Y_{j}\right)=\frac{1}{90}, \quad j=1, \ldots, n .
\end{aligned}
$$

Write

$$
\overline{R_{n}}=\frac{1}{n} \sum_{j=1}^{n} R_{j}
$$

then by the CLT

$$
3 \sqrt{10 n}\left(\overline{R_{n}}-\frac{1}{3}\right) \stackrel{D}{\rightarrow} V \sim N(0,1)
$$

and hence

$$
D_{n}^{(1)}:=45 \cdot 2 n\left(\overline{R_{n}}-\frac{1}{3}\right)^{2} \stackrel{D}{\rightarrow} \chi^{2}(1),
$$

and so $D_{n}^{(1)}$ provides a simple asymptotic test of the hypothesis $X \sim F$.

Setting $X_{j}^{*}=\max \left(X_{2 j-1}, X_{2 j}\right), j=1, \ldots, n$, we note that $D_{n}^{(1)}$ in $(2.1)$ has the form

$$
D_{n}^{(1)}=45 \cdot 2 n\left(\frac{1}{n} \sum_{j=1}^{n} F\left(X_{j}^{*}\right)-\frac{1}{2 n} \sum_{j=1}^{2 n} F^{2}\left(X_{j}\right)-\frac{1}{3}\right)^{2} .
$$

Next we construct goodness-of-fit tests based on the characterization in (1.2) (see also (1.4)), which we write in the form

$$
\operatorname{EF}\left(\max \left(X_{1}, X_{2}\right)\right)=\frac{2}{3}, \quad E F^{2}\left(X_{1}\right)=\frac{1}{3} .
$$

Define

$$
\begin{gathered}
\mathbf{W}_{j}=\left(\begin{array}{c}
Y_{j} \\
Z_{j}
\end{array}\right), \quad j=1, \ldots, n \\
\mu=E \mathbf{W}_{1}=\left(\begin{array}{l}
2 / 3 \\
2 / 3
\end{array}\right)=\frac{2}{3}\left(\begin{array}{l}
1 \\
1
\end{array}\right) \\
\Sigma:=\operatorname{Var}\left(\mathbf{W}_{1}\right)=E\left(\mathbf{W}_{1}-E \mathbf{W}_{1}\right)\left(\mathbf{W}_{1}-E \mathbf{W}_{1}\right)^{\prime}=\left(\begin{array}{ll}
8 / 45 & 4 / 45 \\
4 / 45 & 1 / 18
\end{array}\right)
\end{gathered}
$$

and write $\overline{\mathbf{W}_{n}}=n^{-1} \sum_{j=1}^{n} \mathbf{W}_{j}$. The CLT says that

$$
\sqrt{n}\left(\overline{\mathbf{W}_{n}}-\mu\right) \stackrel{D}{\rightarrow} \mathbf{V} \sim N(0, \Sigma)
$$

whence

$$
D_{n}^{(2)}:=n\left(\overline{\mathbf{W}_{n}}-\mu\right)^{\prime} \Sigma^{-1}\left(\overline{\mathbf{W}_{n}}-\mu\right) \stackrel{D}{\rightarrow} \mathbf{V}^{\prime} \Sigma^{-1} \mathbf{V} \sim \chi^{2}(2) .
$$

But $D_{n}^{(2)}$ is a reasonable measure of the "size" of $\left(\overline{\mathbf{W}_{n}}-\mu\right)$ and so by $(2.3)$ provides a test of the hypothesis that $X$ has the distribution function $F$. And since

$$
\Sigma^{-1}=45\left(\begin{array}{cc}
5 / 8 & -1 \\
-1 & 2
\end{array}\right)
$$


it follows that in extended form

$$
D_{n}^{(2)}=45 n\left[\frac{5}{8}\left(\overline{Y_{n}}-\frac{2}{3}\right)^{2}+2\left(\overline{Z_{n}}-\frac{2}{3}\right)^{2}-2\left(\overline{Y_{n}}-\frac{2}{3}\right)\left(\overline{Z_{n}}-\frac{2}{3}\right)\right] .
$$

In terms of $X_{j}^{*}, D_{n}^{(2)}$ in (2.4) has the form

$$
D_{n}^{(2)}=45 \cdot 2 n\left[\frac{1}{4}\left(\overline{F^{2}\left(X_{2 n}\right)}-\frac{1}{3}\right)^{2}+\left(\overline{F^{2}\left(X_{2 n}\right)}-\overline{F\left(X_{n}^{*}\right)}+\frac{1}{3}\right)^{2}\right] .
$$

By (2.5) and (2.2) we have

LEMMA 2.

$$
D_{n}^{(2)}=\frac{45}{4} \cdot 2 n\left(\overline{F^{2}\left(X_{2 n}\right)}-\frac{1}{3}\right)^{2}+D_{n}^{(1)} .
$$

Special cases:

(a) If $X \sim U(\alpha, \beta)$ then

$$
\begin{aligned}
& D_{n}^{(1)}=45 \cdot 2 n\left(\frac{1}{(\beta-\alpha)^{2}} \overline{X_{2 n}^{2}}-\frac{\beta+\alpha}{(\beta-\alpha)^{2}} \overline{X_{2 n}}-\frac{1}{\beta-\alpha} \overline{X_{n}^{+}}+\frac{\alpha \beta}{\beta-\alpha}+\frac{1}{3}\right)^{2}, \\
& D_{n}^{(2)}=\frac{45}{4} \cdot 2 n\left(\frac{\overline{X_{2 n}^{2}}}{(\beta-\alpha)^{2}}-2 \alpha \frac{\overline{X_{2 n}}}{(\beta-\alpha)^{2}}+\frac{\alpha^{2}}{(\beta-\alpha)^{2}}-\frac{1}{3}\right)^{2}+D_{n}^{(1)} .
\end{aligned}
$$

Remark. If $X \sim U(0, \beta)$ then

$$
\begin{aligned}
& D_{n}^{(1)}=45 \cdot 2 n\left(\overline{X_{2 n}^{2}} / \beta^{2}-\overline{X_{2 n}} / \beta-\overline{X_{n}^{+}} / \beta+\frac{1}{3}\right)^{2}, \\
& D_{n}^{(2)}=\frac{45}{4} \cdot 2 n\left(\overline{X_{2 n}^{2}} / \beta^{2}-\frac{1}{3}\right)^{2}+D_{n}^{(1)} .
\end{aligned}
$$

(b) If $X \sim \operatorname{Pow}(\alpha)$ (power distribution), i.e. $F(x)=1-(1-x / \alpha)^{\alpha}$, $0 \leq x \leq \alpha, 0<\alpha \leq 1$, then

$$
\begin{aligned}
& D_{n}^{(1)}=45 \cdot 2 n\left(\frac{1}{2 n} \sum_{j=1}^{2 n}\left(1-\left(1-X_{j} / \alpha\right)^{\alpha}\right)^{2}+\frac{1}{n} \sum_{j=1}^{n}\left(1-X_{j}^{*} / \alpha\right)^{\alpha}-\frac{2}{3}\right)^{2}, \\
& D_{n}^{(2)}=\frac{45}{4} \cdot 2 n\left(\frac{1}{2 n} \sum_{j=1}^{2 n}\left(1-\left(1-X_{j} / \alpha\right)^{\alpha}\right)^{2}-\frac{1}{3}\right)^{2}+D_{n}^{(1)} .
\end{aligned}
$$

(c) If $X \sim \operatorname{Exp}(\alpha)$ then

$$
\begin{aligned}
& D_{n}^{(1)}=45 \cdot 2 n\left(\frac{1}{2 n} \sum_{j=1}^{2 n}\left(1-e^{-\alpha X_{j}}\right)^{2}+\frac{1}{n} \sum_{j=1}^{n} e^{-\alpha X_{j}^{*}}-\frac{2}{3}\right)^{2}, \\
& D_{n}^{(2)}=\frac{45}{4} \cdot 2 n\left(\frac{1}{2 n} \sum_{j=1}^{2 n}\left(1-e^{-\alpha X_{j}}\right)^{2}-\frac{1}{3}\right)^{2}+D_{n}^{(1)} .
\end{aligned}
$$


(d) If $X \sim W(\beta, \alpha)$ (Weibull distribution), i.e. $F(x)=1-\exp \left(-\alpha x^{\beta}\right)$, $x>0, \alpha>0, \beta>0$, then

$$
\begin{aligned}
& D_{n}^{(1)}=45 \cdot 2 n\left(\frac{1}{2 n} \sum_{j=1}^{2 n}\left(1-e^{-\alpha X_{j}^{\beta}}\right)^{2}+\frac{1}{n} \sum_{j=1}^{n} e^{-\alpha\left(X_{j}^{*}\right)^{\beta}}-\frac{2}{3}\right)^{2}, \\
& D_{n}^{(2)}=\frac{45}{4} \cdot 2 n\left(\frac{1}{2 n} \sum_{j=1}^{2 n}\left(1-e^{-\alpha X_{j}^{\beta}}\right)^{2}-\frac{1}{3}\right)^{2}+D_{n}^{(1)} .
\end{aligned}
$$

(e) If $X \sim \operatorname{Par}_{S}(\alpha, \sigma)$ (single-parameter Pareto distribution), i.e. $F(x)=$ $1-(\sigma / x)^{\alpha}, x>\sigma, \alpha>0, \sigma>0$, then

$$
\begin{aligned}
& D_{n}^{(1)}=45 \cdot 2 n\left(\frac{1}{2 n} \sum_{j=1}^{2 n}\left(1-\left(\frac{\sigma}{X_{j}}\right)^{\alpha}\right)^{2}+\frac{1}{n} \sum_{j=1}^{n}\left(\sigma / X_{j}^{*}\right)^{\alpha}-\frac{2}{3}\right)^{2}, \\
& D_{n}^{(2)}=\frac{45}{4} \cdot 2 n\left(\frac{1}{2 n} \sum_{j=1}^{2 n}\left(1-\left(\frac{\sigma}{X_{j}}\right)^{\alpha}\right)^{2}-\frac{1}{3}\right)^{2}+D_{n}^{(1)} .
\end{aligned}
$$

(f) If $X \sim \operatorname{Par}_{T}(\alpha, \theta)$ (two-parameter Pareto distribution), i.e. $F(x)=$ $1-\left(\frac{\theta}{x+\theta}\right)^{\alpha}, x>0, \alpha>0, \theta>0$, then

$$
\begin{aligned}
& D_{n}^{(1)}=45 \cdot 2 n\left(\frac{1}{2 n} \sum_{j=1}^{2 n}\left(1-\left(\frac{\theta}{X_{j}+\theta}\right)^{\alpha}\right)^{2}+\frac{1}{n} \sum_{j=1}^{n}\left(\frac{\theta}{X_{j}^{*}+\theta}\right)^{\alpha}-\frac{2}{3}\right)^{2}, \\
& D_{n}^{(2)}=\frac{45}{4} \cdot 2 n\left(\frac{1}{2 n} \sum_{j=1}^{2 n}\left(1-\left(\frac{\theta}{X_{j}+\theta}\right)^{\alpha}\right)^{2}-\frac{1}{3}\right)^{2}+D_{n}^{(1)} .
\end{aligned}
$$

(g) If $X \sim \log (\alpha, \beta)$ (logistic distribution), i.e.

$$
F(x)=[1+\exp (-(x-\alpha) / \beta)]^{-1}, \quad-\infty<x<\infty, \alpha \in \mathbb{R}, \beta>0,
$$

then

$$
\begin{aligned}
D_{n}^{(1)}= & 45 \cdot 2 n\left(\frac{1}{2 n} \sum_{j=1}^{2 n}\left(1+\exp \left(-\left(X_{j}-\alpha\right) / \beta\right)\right)^{-2}\right. \\
& \left.-\frac{1}{n} \sum_{j=1}^{n}\left(1+\exp \left(-\left(X_{j}^{*}-\alpha\right) / \beta\right)\right)^{-1}+\frac{1}{3}\right)^{2}, \\
D_{n}^{(2)}= & \frac{45}{4} \cdot 2 n\left(\frac{1}{2 n} \sum_{j=1}^{2 n}\left(1+\exp \left(-\left(X_{j}-\alpha\right) / \beta\right)\right)^{-2}-\frac{1}{3}\right)^{2}+D_{n}^{(1)} .
\end{aligned}
$$

(B) Unknown parameters. We discuss asymptotic tests obtained from $D_{n}^{(1)}$ and $D_{n}^{(2)}$ in (A) when parameters are replaced by estimators. 
Proposition 1. Goodness-of-fit tests for $F(x)=x / \beta, x \in(0, \beta), \beta>0$, are given by

$$
\begin{aligned}
& \widehat{D}_{n}^{(1)}:=D_{n}^{(1)}\left(\widehat{\beta}_{n}\right)=45 \cdot 2 n\left(\frac{\overline{X_{2 n}^{2}}}{\widehat{\beta}_{n}^{2}}-\frac{\overline{X_{2 n}}}{\widehat{\beta}_{n}}-\frac{\overline{X_{n}^{+}}}{\widehat{\beta}_{n}}+\frac{1}{3}\right)^{2} \stackrel{D}{\rightarrow} \chi^{2}(1), \\
& \widehat{D}_{n}^{(2)}:=D_{n}^{(2)}\left(\widehat{\beta}_{n}\right)=\frac{45}{4} \cdot 2 n\left(\frac{\overline{X_{2 n}^{2}}}{\widehat{\beta}_{n}^{2}}-\frac{1}{3}\right)^{2}+D_{n}^{(1)}\left(\widehat{\beta}_{n}\right) \stackrel{D}{\rightarrow} \chi^{2}(2),
\end{aligned}
$$

where $\widehat{\beta}_{n}=\max \left(X_{1}, \ldots, X_{2 n}\right)$.

Proposition 2. Goodness-of-fit tests for $F(x)=\frac{x-\alpha}{\beta-\alpha}, x \in(\alpha, \beta)$, $\alpha<\beta$, are given by

$$
\begin{aligned}
\widehat{D}_{n}^{(1)}:=D_{n}^{(1)}\left(\widehat{\alpha}_{n}, \widehat{\beta}_{n}\right)= & 45 \cdot 2 n\left(\frac{\overline{X_{2 n}^{2}}}{\left(\widehat{\beta}_{n}-\widehat{\alpha}_{n}\right)^{2}}-\left(\widehat{\beta}_{n}+\widehat{\alpha}_{n}\right) \frac{\overline{X_{2 n}}}{\left(\widehat{\beta}_{n}-\widehat{\alpha}_{n}\right)^{2}}\right. \\
& \left.-\frac{\overline{X_{n}^{+}}}{\left(\widehat{\beta}_{n}-\widehat{\alpha}_{n}\right)}+\frac{\widehat{\alpha}_{n} \widehat{\beta}_{n}}{\left(\widehat{\beta}_{n}-\widehat{\alpha}_{n}\right)^{2}}+\frac{1}{3}\right)^{2} \stackrel{D}{\rightarrow} \chi^{2}(1), \\
\widehat{D}_{n}^{(2)}:=D_{n}^{(2)}\left(\widehat{\alpha}_{n}, \widehat{\beta}_{n}\right)= & \frac{45}{4} \cdot 2 n\left(\frac{\overline{X_{2 n}^{2}}}{\left(\widehat{\beta}_{n}-\widehat{\alpha}_{n}\right)^{2}}-2 \frac{\widehat{\alpha}_{n} X_{2 n}}{\left(\widehat{\beta}_{n}-\widehat{\alpha}_{n}\right)}\right. \\
& \left.+\frac{\widehat{\alpha}_{n}^{2}}{\left(\widehat{\beta}_{n}-\widehat{\alpha}_{n}\right)^{2}}-\frac{1}{3}\right)^{2}+D_{n}^{(1)}\left(\widehat{\alpha}_{n}, \widehat{\beta}_{n}\right) \stackrel{D}{\rightarrow} \chi^{2}(2),
\end{aligned}
$$

where $\widehat{\beta}_{n}=\max \left(X_{1}, \ldots, X_{2 n}\right)$ and $\widehat{\alpha}_{n}=\min \left(X_{1}, \ldots, X_{2 n}\right)$.

The proofs of Propositions 1 and 2 are given in [6] and [7]. For the following propositions concerning exponential and normal distributions we use a general theorem based on results in [8] and [9].

Theorem $5([8])$. Let $\widehat{T}_{n}=T_{n}\left(X_{1}, \ldots, X_{n} ; \widehat{\lambda}_{n}\right)$, where $\widehat{\lambda}_{n}=\widehat{\lambda}_{n}\left(X_{1}, \ldots\right.$ $\left.\ldots, X_{n}\right)$ is an estimator of a parameter $\lambda$ of the distribution of $X$, and let $T_{n}=T_{n}\left(X_{1}, \ldots, X_{n} ; \lambda\right)$ (here $T_{n}, \lambda$ and $\widehat{\lambda}_{n}$ may be vectors). Suppose that:

(i) For each $\lambda$,

$$
\sqrt{n}\left(\begin{array}{c}
T_{n} \\
\widehat{\lambda}_{n}-\lambda
\end{array}\right) \stackrel{D}{\rightarrow} T \sim N(0, V)
$$

where

$$
V=\left(\begin{array}{ll}
V_{11} & V_{12} \\
V_{21} & V_{22}
\end{array}\right)
$$

and $V_{22}$ is nonsingular.

(ii) There is a matrix $B$, possibly depending continuously on $\lambda$, such that

$$
\sqrt{n} \widehat{T}_{n}=\sqrt{n} T_{n}+B \sqrt{n}\left(\widehat{\lambda}_{n}-\lambda\right)+o_{p}(1) .
$$


(iii) $\widehat{\lambda}_{n}$ is asymptotically efficient (cf. [8]).

Then

$$
\sqrt{n} \widehat{T}_{n} \stackrel{D}{\rightarrow} T^{*} \sim N\left(0, V_{11}-B V_{22} B^{\prime}\right) .
$$

Note that (ii) is satisfied when $T_{n}$ is differentiable in $\lambda$, and then

$$
B=\lim _{n \rightarrow \infty} E\left[\frac{\partial}{\partial \lambda} T_{n}\right] .
$$

The following result is a consequence of Theorem 5 .

TheOrem 6. Let $\left(X_{1}, \ldots, X_{2 n}\right)$ be a sample with an absolutely continuous distribution function $F(x ; \lambda)$ differentiable with respect to the $m \times 1$ vector $\lambda$. Set

$$
\left.\overline{\mathbf{W}_{n}}:=\left(\overline{Y_{n}}\right):=\left(\overline{Y_{n}(\lambda)}\right)=\overline{Z_{n}(\lambda)}\right),
$$

where

$$
\overline{Y_{n}}=\frac{1}{n} \sum_{j=1}^{2 n} F^{2}\left(X_{j} ; \lambda\right), \quad \overline{Z_{n}}=\frac{1}{n} \sum_{j=1}^{n} F\left(X_{j}^{*} ; \lambda\right),
$$

and $X_{j}^{*}=\max \left(X_{2 j-1}, X_{2 j}\right), j=1, \ldots, n$. Write

$$
\widehat{W}_{n}=\overline{W_{n}\left(\widehat{\lambda}_{2 n}\right)}=\left(\begin{array}{c}
\widehat{Y}_{n} \\
\widehat{Z}_{n}
\end{array}\right),
$$

where

$$
\widehat{Y}_{n}:=\overline{Y_{n}\left(\widehat{\lambda}_{2 n}\right)}, \quad \widehat{Z}_{n}:=\overline{Z_{n}\left(\widehat{\lambda}_{2 n}\right)} .
$$

and $\widehat{\lambda}_{2 n}$ is the MLE of $\lambda$. Suppose that $F$ is such that the MLE $\widehat{\lambda}_{2 n}$ is "regular" in the sense that

$$
\sqrt{2 n}\left(\widehat{\lambda}_{2 n}-\lambda\right) \stackrel{D}{\rightarrow} \gamma \sim N\left(0, I^{-1}\right),
$$

where $I=I(\lambda)$ is the information matrix for $\lambda$ based on a single observation. Then

$$
\begin{gathered}
\sqrt{n}\left(\overline{W_{n}}\left(\widehat{\lambda}_{2 n}\right)-\mu\right) \stackrel{D}{\rightarrow} W \sim N\left(0, \Sigma_{1}\right), \\
\widehat{D}_{n}^{(1)}:=45 \cdot 2 n\left(\overline{\widehat{F}\left(X_{n}^{*}\right)}-\overline{\widehat{F}^{2}\left(X_{2 n}\right)}-\frac{1}{3}\right)^{2} \rightarrow \chi^{2}(1), \\
\widehat{D}_{n}^{(2)}:=\frac{45}{4-b} 2 n\left(\overline{\widehat{F}^{2}\left(X_{2 n}\right)}-\frac{1}{3}\right)^{2}+\widehat{D}_{n}^{(1)} \rightarrow \chi^{2}(2),
\end{gathered}
$$

where $\Sigma_{1}=\Sigma-B(2 I)^{-1} B, \mu$ and $\Sigma$ are taken from $(2.3), \widehat{F}(x):=F(x, \widehat{\lambda})$, and

$$
B=2\left(\begin{array}{l}
2 \\
1
\end{array}\right) d^{\prime},
$$


where

$$
d:=E\left(F(X ; \lambda) \frac{d F(X ; \lambda)}{d \lambda}\right) \quad \text { is } m \times 1 \text {, }
$$

and

$$
b:=b(\lambda)=180 d^{\prime} I^{-1} d .
$$

Proof. The statement (2.7) follows directly from (2.3) and (2.6). Now note that

$$
\begin{aligned}
E \frac{\partial \overline{Y_{n}}}{\partial \lambda_{j}} & =2 E \frac{\partial F^{2}(X ; \lambda)}{\partial \lambda_{j}}=4 E\left(F \frac{\partial F}{\partial \lambda_{j}}\right) \\
& =4 \int F(x ; \lambda) \frac{\partial F}{\partial \lambda_{j}} f(x ; \lambda) d x, \quad j=1, \ldots, m
\end{aligned}
$$

and correspondingly

$$
E\left(\frac{\partial \overline{Z_{n}}}{\partial \lambda_{j}}\right)=E\left(\frac{\partial F\left(\max \left(X_{2 j-1}, X_{2 j}\right) ; \lambda\right)}{\partial \lambda_{j}}\right)=\frac{1}{2} E \frac{\partial \overline{Y_{n}}}{\partial \lambda_{j}}, \quad j=1, \ldots, m,
$$

since the pdf of $X_{i}^{*}=\max \left(X_{2 i-1}, X_{2 i}\right)$ is $2 F\left(x^{*} ; \lambda\right) f\left(x^{*} ; \lambda\right), i=1, \ldots, n$.

It follows that

$$
B=\lim _{n \rightarrow \infty} E\left(\frac{\partial \overline{W_{n}}}{\partial \lambda}\right)=\left(\begin{array}{l}
4 \\
2
\end{array}\right)\left(E\left(F \frac{\partial F}{\partial \lambda_{1}}\right) \ldots E\left(F \frac{\partial F}{\partial \lambda_{m}}\right)\right)=2\left(\begin{array}{l}
2 \\
1
\end{array}\right) d^{\prime}
$$

and hence that

$$
B(2 I)^{-1} B^{\prime}=2 d^{\prime} I^{-1} d\left(\begin{array}{ll}
4 & 2 \\
2 & 1
\end{array}\right)=\frac{b}{90}\left(\begin{array}{ll}
4 & 2 \\
2 & 1
\end{array}\right) .
$$

Thus we have

$$
\begin{aligned}
\Sigma_{1} & =\Sigma-B(2 I)^{-1} B^{\prime}=\frac{1}{90}\left(\begin{array}{cc}
16 & 8 \\
8 & 5
\end{array}\right)-\frac{b}{90}\left(\begin{array}{ll}
4 & 2 \\
2 & 1
\end{array}\right) \\
& =\frac{1}{90}\left(\begin{array}{cc}
4(4-b) & 2(4-b) \\
2(4-b) & 5-b
\end{array}\right),
\end{aligned}
$$

and

$$
\Sigma_{1}^{-1}=90\left(\begin{array}{cc}
5-b /(4(4-b)) & -1 / 2 \\
-1 / 2 & 1
\end{array}\right)
$$

Therefore

$$
\widehat{D}_{n}^{(2)}=n(\widehat{W}-\mu)^{\prime} \Sigma_{1}^{-1}(\widehat{W}-\mu) \stackrel{D}{\rightarrow} \chi^{2}(2),
$$

which (in extended form) proves (2.9).

Finally, writing $a=\left(\begin{array}{c}-1 / 2 \\ 1\end{array}\right)$ we see that

$$
\sqrt{n}\left(\widehat{Z}_{n}-\frac{1}{2} \widehat{Y}_{n}-\frac{1}{3}\right)=a^{\prime}(\sqrt{n}(\widehat{W}-\mu)) \stackrel{D}{\rightarrow} a^{\prime} W \sim N\left(0, a^{\prime} \Sigma_{1} a\right),
$$

and $a^{\prime} \Sigma_{1} a=1 / 90$, which shows (2.8) and completes the proof of Theorem 6 . 
Proposition 3. Goodness-of-fit tests for $X \sim \operatorname{Exp}(\alpha)$ are given by

$$
\begin{aligned}
\widehat{D}_{n}^{(1)} & :=D_{n}^{(1)}\left(\widehat{\alpha}_{2 n}\right) \\
& =45 \cdot 2 n\left(\frac{1}{2 n} \sum_{j=1}^{2 n}\left(1-e^{-\widehat{\alpha}_{2 n} X_{j}}\right)^{2}+\frac{1}{n} \sum_{j=1}^{n} e^{-\widehat{\alpha}_{2 n} X_{j}^{*}}-\frac{2}{3}\right)^{2} \\
& \stackrel{D}{\rightarrow} \chi^{2}(1), \\
\widehat{D}_{n}^{(2)} & :=D_{n}^{(2)}\left(\widehat{\alpha}_{2 n}\right) \\
& =\frac{45 \cdot 36}{19} 2 n\left(\frac{1}{2 n} \sum_{j=1}^{2 n}\left(1-e^{-\widehat{\alpha}_{2 n} X_{j}}\right)^{2}-\frac{1}{3}\right)^{2}+\widehat{D}_{n}^{(1)} \stackrel{D}{\rightarrow} \chi^{2}(2),
\end{aligned}
$$

where $\widehat{\alpha}_{2 n}=1 / \overline{X_{2 n}}$.

Proof. The first statement of Proposition 3 follows from (c) after Lemma 2 and Theorem 6 . To prove the second statement it is enough to see that for $X \sim \operatorname{Exp}(\alpha)$ we have $I(\alpha)=1 / \alpha^{2}$,

$$
d=\alpha \int_{0}^{\infty}\left(x e^{-2 \alpha x}-x e^{-3 \alpha x}\right) d x=5 /(36 \alpha),
$$

and $b=125 / 36$, which by $(2.9)$ gives the test statistic $\widehat{D}_{n}^{(2)}$.

Proposition 4. Goodness-of-fit tests for $X \sim N\left(\mu, \sigma^{2}\right)$ with

$$
\begin{aligned}
& F(x)=\frac{1}{\sqrt{2 \pi} \sigma} \int_{-\infty}^{x} e^{-(t-\mu)^{2} /\left(2 \sigma^{2}\right)} d t, \\
& f(x)=\frac{1}{\sqrt{2 \pi} \sigma} e^{-(x-\mu)^{2} /\left(2 \sigma^{2}\right)}, \quad-\infty<x<\infty,
\end{aligned}
$$

are given by

$$
\begin{aligned}
\widehat{D}_{n}^{(1)} & :=D_{n}^{(1)}\left(\widehat{\mu}_{2 n}, \widehat{\sigma}_{2 n}^{2}\right) \\
& =45 \cdot 2 n\left(\overline{\Phi^{2}\left(\left(X_{2 n}-\widehat{\mu}_{2 n}\right) / \widehat{\sigma}_{2 n}\right)}-\overline{\Phi\left(\left(X_{n}^{*}-\widehat{\mu}_{2 n}\right) / \widehat{\sigma}_{2 n}\right)}+\frac{1}{3}\right)^{2} \\
& \stackrel{D}{\rightarrow} \chi^{2}(1), \\
\widehat{D}_{n}^{(2)} & :=D_{n}^{(2)}\left(\widehat{\mu}_{2 n}, \widehat{\sigma}_{2 n}^{2}\right) \\
& =\frac{45 \cdot 8 \pi^{2}}{32 \pi^{2}-15(6 \pi+1)} \cdot 2 n\left(\overline{\Phi^{2}\left(\left(X_{2 n}-\widehat{\mu}_{2 n}\right) / \widehat{\sigma}_{2 n}\right)}-\frac{1}{3}\right)^{2}+\widehat{D}_{n}^{(1)},
\end{aligned}
$$

where

$$
\Phi(x)=\frac{1}{\sqrt{2 \pi}} \int_{-\infty}^{x} e^{-t^{2} / 2} d t
$$




$$
\begin{aligned}
\overline{\Phi^{2}\left(\left(X_{2 n}-\widehat{\mu}_{2 n}\right) / \widehat{\sigma}_{2 n}\right)} & =\frac{1}{2 n} \sum_{j=1}^{2 n} \Phi^{2}\left(\left(X_{j}-\widehat{\mu}_{2 n}\right) / \widehat{\sigma}_{2 n}\right), \\
\overline{\Phi\left(\left(X_{n}^{*}-\widehat{\mu}_{2 n}\right) / \widehat{\sigma}_{2 n}\right)} & =\frac{1}{n} \sum_{j=1}^{n} \Phi\left(\left(X_{j}^{*}-\widehat{\mu}_{n}\right) / \widehat{\sigma}_{n}\right),
\end{aligned}
$$

and

$$
\widehat{\mu}_{2 n}=\overline{X_{2 n}}, \quad \widehat{\sigma}_{2 n}^{2}=\frac{1}{2 n} \sum_{j=1}^{2 n}\left(X_{j}-\overline{X_{2 n}}\right)^{2} .
$$

Proof. Here

$$
I^{-1}=\left(\begin{array}{cc}
\sigma^{2} & 0 \\
0 & 2 \sigma^{4}
\end{array}\right)
$$

and

$$
\frac{\partial F}{\sigma \mu}=-f, \quad \frac{\partial F}{\partial \sigma^{2}}=-\frac{1}{2 \sigma^{2}}(x-\mu) f,
$$

So

$$
d_{1}=-\int_{-\infty}^{\infty} F(x) f^{2}(x) d x, \quad d_{2}=-\frac{1}{2 \sigma^{2}} \int_{-\infty}^{\infty}(x-\mu) F(x) f^{2}(x) d x
$$

To evaluate the integrals, write

$$
F(x)=\frac{1}{\sqrt{2 \pi}} \int_{-\infty}^{x_{1}} e^{-y^{2} / 2} d y=\frac{1}{2}+\psi\left(x_{1}\right),
$$

where

$$
x_{1}=(x-\mu) / \sigma, \quad \psi\left(x_{1}\right)=\frac{1}{\sqrt{2 \pi}} \int_{0}^{x_{1}} e^{-y^{2} / 2} d y .
$$

Changing variables to $x_{1}=(x-\mu) / \sigma$ gives

$$
d_{1}=-\frac{1}{2 \pi \sigma} \int_{-\infty}^{\infty}\left(\frac{1}{2}+\psi\left(x_{1}\right)\right) e^{-x_{1}^{2}} d x_{1}=-\frac{1}{4 \pi \sigma} \int_{-\infty}^{\infty} e^{-x_{1}^{2}} d x_{1}=-\frac{1}{4 \sqrt{\pi} \sigma},
$$

where we have used the fact that $\psi$ is an odd function. Similarly

$$
\begin{aligned}
d_{2} & =-\frac{1}{4 \pi \sigma^{2}} \int_{-\infty}^{\infty}\left(\frac{1}{2}+\psi\left(x_{1}\right)\right) x_{1} e^{-x_{1}^{2}} d x_{1}=-\frac{1}{4 \pi \sigma^{2}} \int_{-\infty}^{\infty} \psi\left(x_{1}\right) x_{1} e^{-x_{1}^{2}} d x_{1} \\
& =-\frac{1}{8 \pi \sigma^{2}} \int_{-\infty}^{\infty} \psi^{\prime}\left(x_{1}\right) e^{-x_{1}^{2}} d x_{1}=-\frac{1}{8 \sqrt{3} \pi \sigma^{2}},
\end{aligned}
$$

where we have used integration by parts and the facts that $x_{1} e^{-x_{1}^{2}}$ is an odd function and

$$
\psi^{\prime}\left(x_{1}\right)=\frac{1}{\sqrt{2 \pi}} e^{-x_{1}^{2} / 2}
$$


Hence

$$
b=180\left(d_{1}, d_{2}\right)\left(\begin{array}{cc}
\sigma^{2} & 0 \\
0 & 2 \sigma^{4}
\end{array}\right)\left(d_{1}, d_{2}\right)^{\prime}=\frac{15(6 \pi+1)}{8 \pi^{2}},
$$

which by (2.9) leads us to the $\widehat{D}_{n}^{(2)}$ test. Then $\widehat{D}_{n}^{(1)}$ is obtained immediately from (2.8).

3. Goodness-of-fit tests based on characterizations via moments of record values. Suppose that $X$ has df $F$ and pdf $f$. To simplify the notation we write

$$
g(x)=1-F(x) \quad \text { and } \quad h(x)=-\log (g(x))
$$

if $F(x)<1$ and 0 otherwise.

Then Theorem 3 says (see (1.5)) that $X \sim F$ iff

$$
k^{2 l}(n-l) ! E h^{2 l}\left(Y_{n-l+1}^{(k)}\right)-2 n ! k^{l} E h^{l}\left(Y_{n+1}^{(k)}\right)+(n+l) !=0 .
$$

Since the definition of $Y_{n}^{(k)}$ requires an infinite sequence it is hard to see how a finite sample can be used to estimate $E Y_{n}^{(k)}$. So our procedure is as follows.

We consider the special case $l=n$. Then $X \sim F$ iff

$$
E h^{2 n}\left(X_{1: k}\right)-\frac{2 n !}{k^{n}} E h^{n}\left(Y_{n+1}^{(k)}\right)+\frac{(2 n) !}{k^{2 n}}=0 .
$$

We know that the pdf of $Y_{n}^{(k)}$ is

$$
f_{Y_{n}^{(k)}}(x)=\frac{k^{n}}{(n-1) !} h^{n-1}(x) g^{k-1}(x) f(x) \quad(\text { cf. [1]) }
$$

and that

$$
\begin{aligned}
F_{Y_{n+1}^{(k)}}(x) & =F_{Y_{n}^{(k)}}(x)-\frac{k^{n}}{n !} h^{n}(x) g^{k}(x) \\
& =1-g^{k}(x) \sum_{j=0}^{n} \frac{k^{j}}{j !} h^{j}(x) \quad(\text { cf. [2]). }
\end{aligned}
$$

Hence

$$
\begin{aligned}
E h^{n}\left(Y_{n+1}^{(k)}\right)= & E h^{n}\left(Y_{n}^{(k)}\right)-\frac{k^{n}}{(n-1) !} E h^{2 n-1}(X) g^{k-1}(X) \\
& +\frac{k^{n+1}}{n !} E h^{2 n}(X) g^{k-1}(X) .
\end{aligned}
$$

Taking into account that

$$
E g^{\alpha-1}(X) h^{\beta-1}(X)=\frac{\Gamma(\beta)}{\alpha^{\beta}} \quad \text { for } \alpha, \beta>0
$$


as $X$ has df $F$, we get

$$
E h^{n}\left(Y_{n+1}^{(k)}\right)=E h^{n}\left(Y_{n}^{(k)}\right)+\frac{(2 n) !}{2 n ! k^{n}} .
$$

Hence by (3.1) we obtain

$$
E h^{2 n}\left(X_{1: k}\right)-\frac{2 n !}{k^{n}} E h^{n}\left(Y_{n}^{(k)}\right)=0 .
$$

Letting $n=1$ we have

$$
E h^{2}\left(X_{1: k}\right)-\frac{2}{k} E h\left(X_{1: k}\right)=0 .
$$

Similarly using the second equality in (3.2) we get

$$
E h^{2 n}\left(X_{1: k}\right)-\frac{2 n !}{k^{n}} E h^{n}\left(X_{1: k}\right)-\frac{(2 n) !-2(n !)^{2}}{k^{2 n}}=0 .
$$

To verify $H: X \sim F$ we use (3.3). Consider first the case $k=1$. Then

$$
E\left(h^{2}\left(X_{1}\right)-2 h\left(X_{1}\right)\right)=0 \text {. }
$$

The sample $\left(X_{1}, \ldots, X_{n}\right)$ provides an estimator of $E W_{1}$, where $W_{1}=h^{2}\left(X_{1}\right)$ $-2 h\left(X_{1}\right)$, of the form

$$
\overline{W_{n}}=\overline{h^{2}\left(X_{n}\right)}-2 \overline{h\left(X_{n}\right)}
$$

where

$$
\overline{h^{2}\left(X_{n}\right)}=\frac{1}{n} \sum_{j=1}^{n} h^{2}\left(X_{j}\right), \quad \overline{h\left(X_{n}\right)}=\frac{1}{n} \sum_{j=1}^{n} h\left(X_{j}\right) .
$$

It follows from the CLT that

$$
\sqrt{n} \overline{W_{n}} \stackrel{D}{\rightarrow} N\left(0, \operatorname{Var}\left(W_{1}\right)\right)
$$

and hence that

$$
T_{n}^{(1)}:=n{\overline{W_{n}}}^{2} / \operatorname{Var}\left(W_{1}\right) \stackrel{D}{\rightarrow} \chi^{2}(1),
$$

and so provides a simple asymptotic test of the hypothesis $X \sim F$ when the parameters of $F$ are known. Here

$$
\operatorname{Var} W_{1}=E h^{4}\left(X_{1}\right)-4 E h^{3}\left(X_{1}\right)+4 E h^{2}\left(X_{1}\right)=8
$$

since $h\left(X_{1}\right) \sim \operatorname{Exp}(1)$ gives $E h^{m}\left(X_{1}\right)=m !, m=1,2, \ldots$, and so

$$
T_{n}^{(1)}=\frac{n}{8}\left(\overline{h^{2}\left(X_{n}\right)}-2 \overline{h\left(X_{n}\right)}\right)^{2} .
$$

We have proved

Proposition 5. If $X_{n} \sim F, n \geq 1$, are independent then

$$
T_{n}^{(1)}=\frac{n}{8}\left(\overline{h^{2}\left(X_{n}\right)}-2 \overline{h\left(X_{n}\right)}\right)^{2} \stackrel{D}{\rightarrow} \chi^{2}(1) .
$$


Now consider the case $k=2$. Write $U_{1}:=X_{1: 2}=\min \left(X_{1}, X_{2}\right)$. Here from (3.3) we have to estimate $E W_{1}^{\prime}$, where $W_{1}^{\prime}=h^{2}\left(U_{1}\right)-h\left(U_{1}\right)$. The sample $X_{1}, \ldots, X_{2 n}$ provides the sample $W_{1}^{\prime}, \ldots, W_{n}^{\prime}$, where $W_{j}^{\prime}=h^{2}\left(U_{j}\right)-h\left(U_{j}\right)$ and $U_{j}=\min \left(X_{2 j-1}, X_{2 j}\right), j=1, \ldots, n$. Then $E W_{1}^{\prime}$ is estimated by

$$
\overline{W_{n}^{\prime}}=\overline{h^{2}\left(U_{n}\right)}-\overline{h\left(U_{n}\right)},
$$

and

$$
T_{n}^{(2)}:=n\left(\overline{W_{n}^{\prime}}\right)^{2} / \operatorname{Var}\left(W_{1}^{\prime}\right) \stackrel{D}{\rightarrow} \chi^{2}(1) .
$$

Taking into account that $h\left(U_{1}\right) \sim \operatorname{Exp}(2)$ we see that $\operatorname{Var}\left(W_{1}^{\prime}\right)=1 / 2$. Thus another simple asymptotic test is provided by

Proposition 6. If $X_{n} \sim F, n \geq 1$, are independent then

$$
T_{n}^{(2)}=2 n\left(\overline{h^{2}\left(U_{n}\right)}-\overline{h\left(U_{n}\right)}\right)^{2} \stackrel{D}{\rightarrow} \chi^{2}(1) .
$$

The same argument leads to a similar test for the case $k=3, \ldots, n-1$ based on a sample of size $k n$.

We now consider the case $k=n$. Write $U_{n}=\min \left(X_{1}, \ldots, X_{n}\right)$. Then by (3.3) we have to estimate $E\left(h^{2}\left(U_{n}\right)-(2 / n) h\left(U_{n}\right)\right)$. The obvious estimate is $h^{2}\left(U_{n}\right)-(2 / n) h\left(U_{n}\right)$ itself, and if the parameters of $F$ are specified the test statistic is

$$
T_{n}^{(n)}:=\left(h^{2}\left(U_{n}\right)-\frac{2}{n} h\left(U_{n}\right)\right)^{2} .
$$

As above, under $H, h\left(U_{n}\right) \sim \operatorname{Exp}(n)$, whence

$$
R_{n}:=n h\left(U_{n}\right) \sim U \sim \operatorname{Exp}(1), \quad n \geq 1 .
$$

It follows that

$$
T_{n}^{(n)}=\frac{1}{n^{4}}\left(R_{n}^{2}-2 R_{n}\right)^{2}
$$

and so an equivalent test statistic is $T_{n}:=\left(R_{n}^{2}-2 R_{n}\right)^{2} \sim T:=\left(U^{2}-2 U\right)^{2}$, $n \geq 1$, which provides an exact test for $H: X \sim F$.

Proposition 7 (cf. [7]). The significance probability of the test using $T_{n}$ is

$$
\begin{aligned}
P_{t} & :=P\left[T_{n}>t\right] \\
& = \begin{cases}e^{-1-\sqrt{1+\sqrt{t}}}+e^{-1+\sqrt{1-\sqrt{t}}}-e^{-1-\sqrt{1-\sqrt{t}}} & \text { if } 0<t \leq 1, \\
e^{-1-\sqrt{1+\sqrt{t}}} & \text { if } t \geq 1 .\end{cases}
\end{aligned}
$$

Proof. The significance probability $P\left[T_{n}>t\right]$ associated with an observed value $t$ can be obtained by considering the graph of $u^{2}(u-2)^{2}=t$ and using the fact that $P[U<u]=1-e^{-u}$. One finds readily that (3.8) holds true. 
In particular we consider the $5 \%$ test of $H$, i.e. $P_{t}=0.05$. But since

$$
P[T>1]=e^{-(1+\sqrt{2})}>0.05
$$

the $5 \%$ test rejects when $R_{n}>u_{0}$, where $e^{-u_{0}}=0.05$, i.e. when $u_{0}=3.00$. Thus the exact $5 \%$ test rejects when $n h\left(U_{n}\right)>3$.

Now we show that instead of $T_{n}=\left[R_{n}^{2}-2 R_{n}\right]^{2}$ one can use more generally the statistics

$$
T_{n}^{[m]}:=\left\{\left(R_{n}^{m}-m !\right)^{2}-\left((2 m) !-(m !)^{2}\right)\right\}^{2}, \quad m \geq 1 .
$$

We note that $T_{n}=T_{n}^{[1]}$.

Writing (3.4) in the form

$$
E k^{2 m} h^{2 m}\left(X_{1: k}\right)-2 m ! E k^{m} h^{m}\left(X_{1: k}\right)-\left((2 m) !-2(m !)^{2}\right)=0
$$

and letting $k=n$ (sample size), we have

$$
E\left\{\left(\left(n h\left(X_{1: n}\right)\right)^{m}-m !\right)^{2}-\left((2 m) !-(m !)^{2}\right)\right\}=0 .
$$

Taking into account that $R_{n}=n h\left(X_{1: n}\right) \sim \operatorname{Exp}(1), n \geq 1$, we see that

$$
T_{n}^{[m]}=\left\{\left(R_{n}^{m}-m !\right)^{2}-a_{m}\right\}^{2} \sim\left[\left(U^{m}-m !\right)^{2}-a_{m}\right]^{2}
$$

where

$$
a_{m}=(2 m) !-(m !)^{2} .
$$

It follows that the statistics $T_{n}^{[m]}$ have for every $n \geq 1$ the distribution of $\left[\left(U^{m}-m !\right)^{2}-a_{m}\right]^{2}$, and we reject $H: X \sim F$ if $T_{n}^{[m]}$ is large enough. Moreover, we can state the following result.

Proposition 8. The significance probability of the test using $T_{n}^{[m]}$ is

$$
\begin{aligned}
P_{t}^{[m]} & :=P\left[T_{n}^{[m]}>t\right] \\
& = \begin{cases}1-e^{-b_{m}^{(2)}(t)}+e^{-b_{m}^{(3)}(t)} & \text { if } 0<t \leq t_{m}, \\
e^{-b_{m}^{(1)}(t)}-e^{-b_{m}^{(2)}(t)}+e^{-b_{m}^{(3)}(t)} & \text { if } t_{m}<t \leq t_{m}^{\prime}, \\
e^{-b_{m}^{(3)}(t)} & \text { if } t>t_{m}^{\prime}\end{cases}
\end{aligned}
$$

where

$$
\begin{aligned}
& b_{m}^{(1)}(t)=\left(m !-\sqrt{a_{m}-\sqrt{t}}\right)^{1 / m}, \quad b_{m}^{(2)}(t)=\left(m !+\sqrt{a_{m}-\sqrt{t}}\right)^{1 / m}, \\
& b_{m}^{(3)}(t)=\left(m !+\sqrt{a_{m}+\sqrt{t}}\right)^{1 / m}, \quad t_{m}=\left(a_{m}-(m !)^{2}\right)^{2}, \quad t_{m}^{\prime}=a_{m}^{2} .
\end{aligned}
$$

The proof of (3.9) is similar to the proof of Proposition 7. 
Corollary. $P_{t}^{[1]}$ is given by (3.8) and $P_{t}^{[2]}$ is given by the formula

$$
\begin{aligned}
& P_{t}^{[2]}=P\left[T_{n}^{[2]}>t\right]
\end{aligned}
$$

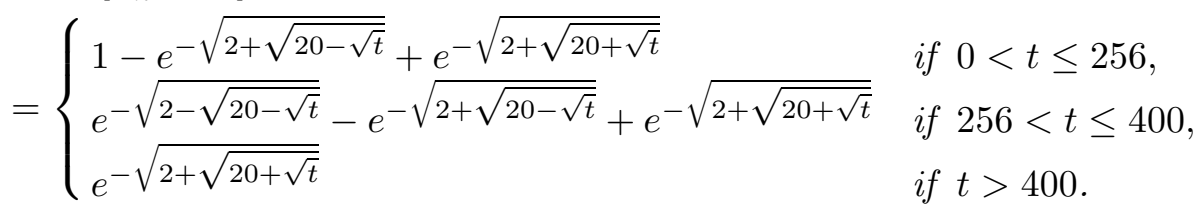

4. Tests for exponentiality. We consider corresponding tests for $X \sim \operatorname{Exp}(\alpha)$ when $\alpha$ is not specified. Note that in this case $h(x)=$ $-\log (1-F(x))=\alpha x$. Using $T_{n}^{(1)}:=T_{n}^{(1)}(\alpha), T_{n}^{(2)}:=T_{n}^{(2)}(\alpha)$ in $(3.5)$ and (3.6) respectively, we replace $\alpha$ by the estimator $\widehat{\alpha}_{n}$. We have proved in [7] the following results.

Proposition 9. If $X_{n} \sim F, n \geq 1$, are independent then

$$
\widehat{T}_{n}^{(1)}:=2 T_{n}^{(1)}\left(\widehat{\alpha}_{n}\right)=\frac{n}{4}\left(\overline{X_{n}^{2}} /\left(\overline{X_{n}}\right)^{2}-2\right)^{2} \stackrel{D}{\rightarrow} \chi^{2}(1),
$$

where $\widehat{\alpha}_{n}=1 / \overline{X_{n}}$.

Proposition 10. If $X_{n} \sim F, n \geq 1$, are independent then

$$
\widehat{T}_{n}^{(2)}:=\frac{4}{3} T_{n}^{(2)}\left(\widehat{\alpha}_{n}\right)=\frac{8}{3}\left(\overline{U_{n}^{2}}-\frac{1}{\widehat{\alpha}_{n}} \overline{U_{n}}\right)^{2}=\frac{8 n}{3}\left(\frac{\overline{U_{n}^{2}}}{\left(\overline{X_{2 n}}\right)^{2}}-\frac{\overline{U_{n}}}{\overline{X_{2 n}}}\right)^{2} \stackrel{D}{\rightarrow} \chi^{2}(1),
$$

where $\widehat{\alpha}_{n}=1 / \overline{X_{2 n}}$.

Proposition 11. Let $\widehat{T}_{n}:=T\left(\widehat{\alpha}_{n}\right)=\left(\widehat{U}_{n}^{2}-2 \widehat{U}_{n}\right)^{2}$ where $\widehat{U}_{n}=n \widehat{\alpha}_{n} U_{n}=$ $n U_{n} / \bar{X}_{n}$, and let $\widehat{P}_{t}:=P\left[\widehat{T}_{n}>t\right]$ stand for the associated significance probability. Then $\lim _{n \rightarrow \infty} \widehat{P}_{t}=P_{t}$, where $P_{t}$ is given by Proposition 7 .

Now by Proposition 8 we have the following generalization of Proposition 11 .

Proposition 12. Let

$$
\widehat{T}_{n}^{[m]}:=T_{n}^{[m]}\left(\widehat{\alpha}_{n}\right)=\left\{\left[\left(n \widehat{\alpha}_{n} U_{n}\right)^{m}-m !\right]^{2}-a_{m}\right\}^{2}
$$

and let $\widehat{P}_{t}^{[m]}:=P\left[\widehat{T}_{n}^{[m]}>t\right]$ stand for the associated significance probability. Then

$$
\lim _{n \rightarrow \infty} \widehat{P}_{t}^{[m]}=P_{t}^{[m]}, \quad m \geq 1
$$

where $P_{t}^{[m]}$ is given by Proposition 8 .

Proof. Since $\widehat{\alpha}_{n} \stackrel{P}{\rightarrow} \alpha$, from (3.7) we get $n \widehat{\alpha}_{n} U_{n}=\left(\widehat{\alpha}_{n} / \alpha\right) R_{n} \stackrel{D}{\rightarrow} U$ and so

$$
\widehat{T}_{n}^{[m]} \stackrel{D}{\rightarrow}\left[\left(U^{m}-m !\right)^{2}-a_{m}\right]^{2},
$$

which is distributed as $T_{n}^{[m]}$. 
Acknowledgements. The authors are very grateful to the referee for a number of useful suggestions.

\section{References}

[1] W. Dziubdziela and B. Kopociński, Limiting properties of the $k$-th record values, Zastos. Mat. 15 (1976), 187-190.

[2] Z. Grudzien and D. Szynal, On the expected values of $k$-th record values and associated characterizations of distributions, in: Proc. 4th Pannonian Symp. on Math. Statist. (Bad Tatzmannsdorf, 1985), 119-127.

[3] - - C, Characterization of continuous distributions in terms of moments of extremal statistics, J. Math. Sci. 81 (1996), 2912-2936.

[4] - - - Characterizations of continuous distributions via moments of record values, J. Appl. Statist. Soc. 9 (2000), 93-103.

[5] G. D. Lin, Characterizations of continuous distributions via expected values of two functions of order statistics, Sankhyā Ser. A 52 (1990), 84-90.

[6] K. Morris and D. Szynal, A goodness-of-fit test for the uniform distribution based on a characterization, J. Math. Sci., submitted.

[7] - - - Goodness-of-fit tests based on characterizations of continuous distributions, Appl. Math. (Warsaw) 27 (2000), 475-488.

[8] D. A. Pierce, The asymptotic effect of substituting estimators for parameters in certain types of statistics, Ann. Statist. 10 (1982), 475-478.

[9] R. H. Randles, On the asymptotic normality of statistics with estimated parameters, ibid., 462-474.

[10] Y. H. Too and G. D. Lin, Characterizations of uniform and exponential distributions, Statist. Probab. Lett. 7 (1989), 357-359.

Department of Applied Mathematics

University of Adelaide

North Tce, Adelaide

South Australia, 5001

E-mail: kmorris@stats.adelaide.edu.au
Institute of Mathematics Maria Curie-Skłodowska University Pl. M. Curie-Skłodowskiej 1 20-031 Lublin, Poland

E-mail: szynal@golem.umcs.lublin.pl

Received on 1.9.2000;

revised version on 26.1.2001 